\title{
The role of glucagon-like peptide-1 receptor agonists in cardiovascular disease prevention in type 2 diabetes mellitus: evidence from the most recent clinical trials
}

\author{
S. John Weisnagel \\ Department of Endocrinology and Nephrology, Faculty of Medicine, Diabetes Research Unit, CHU Research Centre, Laval University, Québec, \\ Canada \\ Correspondence to: S. John Weisnagel, MD, FRCPC. Department of Endocrinology and Nephrology, Faculty of Medicine, Diabetes Research Unit, \\ CHU Research Centre, Laval University, Endocrinologie, B0027, CHUL, CHU de Québec, 2705 Laurier, Québec, G1V 4G2 QC, Canada. \\ Email: John.weisnagel@crchul.ulaval.ca. \\ Provenance: This is an invited Editorial commissioned by Section Editor Kaiping Zhang (AME College, AME Group, Hangzhou, China). \\ Comment on: Holman RR, Bethel MA, Mentz RJ, et al. Effects of Once-Weekly Exenatide on Cardiovascular Outcomes in Type 2 Diabetes. N Engl J \\ Med 2017;377:1228-39.
}

Submitted Feb 18, 2018. Accepted for publication Mar 13, 2018.

doi: $10.21037 /$ atm.2018.03.29

View this article at: http://dx.doi.org/10.21037/atm.2018.03.29

The field of investigation of the prevention of cardiovascular disease (CVD) in type 2 diabetes mellitus (T2DM) has seen a huge proliferation of studies on multiple drug categories and agents in the past decade. Following the controversy around the possible effect of the thiazolidinedione rosiglitazone on CVD events (1), regulatory agencies in the USA (Food and Drug Administration), Europe (European Medicines Agency) and elsewhere have required that all new anti-diabetic agents (ADA) undergo formal evaluation for CVD safety through large multicenter randomised placebo-controlled clinical trials (2). This has led to numerous studies in tens of thousands of patients with T2DM followed for several years, tremendously advancing knowledge in this field, as well as providing extremely valuable information on safety and side effect profiles of the agents tested.

Three newer ADA classes that have emerged in the treatment of T2DM have interesting clinical profiles. Dipeptidyl peptidase 4 (DPP-4) inhibitors are incretins that stimulate glucose-dependant insulin secretion, stimulate glucose-dependant insulin secretion and suppress glucagon secretion, leading to mild decreases in HbAlc with little risk of hypoglycemia, weight neutral effects and possible lowering of blood pressure (3). Glucagon-like peptide-1 (GLP-1) receptor agonist are also incretins that stimulate glucose-dependant insulin secretion, stimulate glucose- dependant insulin secretion and inhibit glucagon secretion, however they cause substantial lowering in HbA1c, body weight and blood pressure (3). Finally, sodium-glucose cotransporter 2 (SGLT-2) inhibitors cause an increase in urinary glucose and sodium excretion, also leading to lowering in $\mathrm{HbAlc}$, body weight and blood pressure (4).

All DPP-4 inhibitors have been tested in large CVD trials, three of which (alogliptin, saxagliptin, and sitagliptin) have been published and showed no effect on major adverse cardiovascular events (MACE) (5-7). Studies in GLP-1 receptor agonists have produced varying results. The exendin-4-based GLP-1 receptor agonist lixisenatide was neutral on CVD outcomes (8), but a lower risk of MACE was shown with the two GLP-1 receptor agonists liraglutide and semaglutide $(9,10)$. As to the SGLT-2 inhibitors empagliflozin and canagliflozin, two recent trials also demonstrated lower MACE in subjects treated with these agents $(11,12)$.

In September 2017, in the New England Fournal of Medicine, the EXSCEL Study Group reported on the results of the large clinical trial $(n=14,752)$ evaluating the effects of a once-weekly subcutaneous injection of extendedrelease exenatide at a dose of $2 \mathrm{mg}$ on CVD outcomes (13). The primary outcome was the traditional three-component MACE defined as death from cardiovascular causes, nonfatal myocardial infarction, or nonfatal stroke. This was 
mainly a secondary prevention trial with $73 \%$ of subjects having had previous CVD. During a median follow-up of 3.2 years, a primary composite outcome event occurred in $11.4 \%$ ( 3.7 events per 100 person-years) in the exenatide group and in $12.2 \%$ (4.0 events per 100 person-years) in the placebo group [hazard ratio $(\mathrm{HR})=0.91 ; 95 \%$ confidence interval (CI), 0.83-1.00]. Noninferiority was attained $(\mathrm{P}<0.001)$ but not superiority $(\mathrm{P}=0.06)$ with respect to efficacy of exenatide.

As expected with the use of a GLP-1 receptor agonist, improvements in several CVD risk factors occurred in the extended-release exenatide group compared to the placebo group, with overall least-squares mean differences of $-0.53 \%$ for $\mathrm{HbA1c},-1.27 \mathrm{~kg}$ for body weight and $-1.57 \mathrm{mmHg}$ for systolic blood pressure. However, heart rate was increased 2.5 beats per minute in the treatment group. Of note, the risk of death from any cause was $6.9 \%$ in the exenatide group and $7.9 \%$ in the placebo group, although this difference was not considered to be statistically significant in per-protocol hierarchical secondary end-point analyses. In univariate analyses in prespecified subgroups defined by baseline characteristics, only the age subgroup (baseline age $<65 v s . \geq 65$ years) showed heterogeneity, with the older group showing benefit $(\mathrm{HR}=0.80)$, and the younger group no effect (HR =1.05). Serious adverse outcomes were similar between groups, in particular with respect to the incidence of acute pancreatitis and pancreatic cancer.

The rate of premature discontinuation of the trial regimen, driven primarily by patient decision, was a major limitation of the trial. The authors speculated that probable causal factors for this were the complexity of the first-generation injection device that was used and the fact that the trial had no run-in period. This resulted in a decrease in the duration of time that participants received the trial regimen relative to the expected duration time (approximately $75.0 \%$, with a median duration of exposure to the trial regimen of 2.3-2.4 years). This may have contributed to the lack of efficacy.

In other CVD prevention studies with GLP-1 receptor agonists, treatment with liraglutide (LEADER trial) and semaglutide (SUSTAIN-6 trial) vs. placebo was associated with lower risk of a three component MACE $(\mathrm{HR}=0.87$; 95\% CI, 0.78-0.97 in LEADER, 0.74; 95\% CI, 0.58-0.95 in SUSTAIN-6). In these studies, the risks for the different individual components of the MACE varied. In the LEADER trial, the hazard ratio for risk of death from any cause was 0.85 with liraglutide vs placebo (similar to the
0.86 seen in the EXSCEL trial), but no such difference was seen with semaglutide in SUSTAIN-6. However, in that study, there was a major effect on non-fatal stroke, with a $\mathrm{HR}=0.61$ in the semaglutide group. Finally, as already mentioned, the GLP-1 receptor agonist lixisenatide was neutral on CVD outcomes.

In a very recent meta-analysis of all CVD trials with GLP-1 receptor agonists, the overall relative risk reduction (RRR) was significant at $10 \%$ for the three-point MACE $(\mathrm{HR}=0.90,95 \% \mathrm{CI}, 0.82-0.99 ; \mathrm{P}=0.033), 13 \%$ for cardiovascular mortality (HR $=0.87,95 \%$ CI, 0.79-0.96; $\mathrm{P}=0.007)$ and $12 \%$ for all-cause mortality ( $\mathrm{HR}=0.88,95 \%$ CI, 0.81-0.95; $\mathrm{P}=0.002)$, with between-trial statistical heterogeneity being low-to-moderate (14). Of course, the basic premise of meta-analyses is that the agents studied are considered to have a similar or "class" effect. This is not yet clear from the data.

When comparing the results from the published studies to date, we can only speculate on the differences in results for the primary MACE and various secondary outcomes. Overall, these were mostly secondary prevention trials, with between $73 \%$ and $83 \%$ of subjects having had a previous CVD event. Therefore, it is not at all certain that patients with T2DM but no previous CVD would benefit from either GLP-1 agonist with respect to MACE. In fact, when examining the results in various prespecified subgroups, the difference from placebo for the MACE outcomes is often minimal in subjects with no previous CVD. As to population characteristics and trial conduct, there were differences in the baseline mean HbA1c in these trials, being higher at approximately $8.7 \%$ in both LEADER and SUSTAIN-6, compared to 8\% in EXSCEL. Also, average exposures to trial drug differed among studies, being lower at $75 \%$ in EXSCEL, but $83-84 \%$ in LEADER and $86.5-89.5 \%$ in SUSTAIN-6. These factors may have influenced the outcomes in EXSCEL, since subjects had better initial glucose control and potential glucose benefits on CVD were thus minimized. Also, the relative underexposure to the GLP-1 receptor agonist may have led to an underestimation of its effect. Finally, in the placebo group, more subjects were treated during the trial with a SGLT2 inhibitor, a drug class known to have favorable CVD effects. This could also have minimized the difference in CVD event rates between the placebo and extended-release exenatide groups.

It is also important to point out that the various GLP-1 receptor agonists have different structures and halflives, which could possibly lead to varying clinical effects. 
Liraglutide and semaglutide have greater homology with native GLP-1. Could this lead to a stronger CVD effect? We could only speculate. Other CVD trials are still underway, particularly with dulaglutide (REWIND) (15) and albiglutide (HARMONY) (16). The results from all these different studies will possibly allow us to conclude on class effects or specific agent effects. Although the primary outcome of MACE in the EXSCEL trial was not significant for superiority, the results did follow the same direction as those of the LEADER and SUSTAIN-6 studies, suggesting a possible class effect.

It is also important to note that the EXSCEL data adds essential information with regard to safety of GLP-1 receptor agonists, almost doubling the number of patients exposed to this ADA class in large randomized placebocontrolled clinical trials. No significant signal was seen for serious adverse events.

Finally, how do these results relate to those seen in studies on other drug classes, such as the SGLT2 inhibitors? Both empagliflozin (EMPA-REG) and canagliflozin (CANVAS) showed benefit for the same MACE outcomes ( $\mathrm{HR}=0.86$ ), with additional positive effects on cardiac failure ( $\mathrm{HR}=0.65-$ 0.67), and relatively strong reductions in CVD mortality (empagliflozin, HR $=0.62$ ). In these studies, the majority of subjects had previous CVD (secondary prevention). Would the combination of a GLP-1 receptor agonist with a SGLT2 inhibitor provide additional benefit? These questions are being actively raised by investigators (17). Could some patients respond better to one class compared to another?

Only very large clinical trials could answer these important questions. Will these occur in the next decade? The diabetes community awaits further developments with anticipation...

\section{Acknowledgements}

None.

\section{Footnote}

Conflicts of Interest: The author was a clinical trial investigator in several of the above mentioned studies. In particular, he participated in or is still involved in the following: TECOS (sitagliptin by Merck), LEADER (liraglutide by Novo-Nordisk), REWIND (dulaglutide by Eli Lilly), DECLARE (dapagliflozin by Astra Zeneca).

\section{References}

1. Nissen SE, Wolski K. Effect of rosiglitazone on the risk of myocardial infarction and death from cardiovascular causes. N Engl J Med 2007;356:2457-71.

2. FDA. Guidance for industry: diabetes mellitus—evaluating cardiovascular risk in new antidiabetic therapies to treat type 2 diabetes. Washington, DC: US Department of Health and Human Resources, 2008.

3. Nauck MA, Meier JJ. Incretin hormones: Their role in health and disease. Diabetes Obes Metab 2018;20 Suppl 1:5-21.

4. Steen O, Goldenberg RM. The Role of Sodium-Glucose Cotransporter 2 Inhibitors in the Management of Type 2 Diabetes. Can J Diabetes 2017;41:517-23.

5. Scirica BM, Bhatt DL, Braunwald E, et al. Saxagliptin and cardiovascular outcomes in patients with type 2 diabetes mellitus. N Engl J Med 2013;369:1317-26.

6. White WB, Cannon CP, Heller SR, et al. Alogliptin after acute coronary syndrome in patients with type 2 diabetes. N Engl J Med 2013;369:1327-35.

7. Green JB, Bethel MA, Armstrong PW, et al. Effect of Sitagliptin on Cardiovascular Outcomes in Type 2 Diabetes. N Engl J Med 2015;373:232-42.

8. Pfeffer MA, Claggett B, Diaz R, et al. Lixisenatide in Patients with Type 2 Diabetes and Acute Coronary Syndrome. N Engl J Med 2015;373:2247-57.

9. Marso SP, Daniels GH, Brown-Frandsen K, et al. Liraglutide and Cardiovascular Outcomes in Type 2 Diabetes. N Engl J Med 2016;375:311-22.

10. Marso SP, Bain SC, Consoli A, et al. Semaglutide and Cardiovascular Outcomes in Patients with Type 2 Diabetes. N Engl J Med 2016;375:1834-44.

11. Zinman B, Wanner C, Lachin JM, et al. Empagliflozin, Cardiovascular Outcomes, and Mortality in Type 2 Diabetes. N Engl J Med 2015;373:2117-28.

12. Mahaffey KW, Neal B, Perkovic V, et al. Canagliflozin for Primary and Secondary Prevention of Cardiovascular Events: Results From the CANVAS Program (Canagliflozin Cardiovascular Assessment Study). Circulation 2018;137:323-34.

13. Holman RR, Bethel MA, Mentz RJ, et al. Effects of OnceWeekly Exenatide on Cardiovascular Outcomes in Type 2 Diabetes. N Engl J Med 2017;377:1228-39.

14. Bethel MA, Patel RA, Merrill P, et al. Cardiovascular outcomes with glucagon-like peptide-1 receptor agonists in patients with type 2 diabetes: a meta-analysis. Lancet Diabetes Endocrinol 2018;6:105-13. 
15. Gerstein HC, Colhoun HM, Dagenais GR, et al. Design and baseline characteristics of participants in the Researching cardiovascular Events with a Weekly INcretin in Diabetes (REWIND) trial on the cardiovascular effects of dulaglutide. Diabetes Obes Metab 2018;20:42-9.

16. Ahrén B, Carr MC, Murphy K, et al. Albiglutide for the treatment of type 2 diabetes mellitus: An integrated safety analysis of the HARMONY phase 3 trials. Diabetes Res Clin Pract 2017;126:230-9.

17. DeFronzo RA. Combination therapy with GLP-1 receptor agonist and SGLT2 inhibitor. Diabetes Obes Metab 2017;19:1353-62.

Cite this article as: Weisnagel SJ. The role of glucagon-like peptide-1 receptor agonists in cardiovascular disease prevention in type 2 diabetes mellitus: evidence from the most recent clinical trials. Ann Transl Med 2018;6(10):194. doi: 10.21037/ atm.2018.03.29 